\title{
The Usage of Fly Ash for Reclaimed Asphalt Pavement (RAP), Asphalt Concrete Binder Course (AC-BC) Filler
}

\author{
Ratna Handayani ${ }^{1}$, Ria Asih Aryani Soemitro ${ }^{1}$, Herry Budianto ${ }^{1}$, and Januarti Jaya Ekaputri ${ }^{1}$
}

\begin{abstract}
Material Reclaimed Asphalt Pavement (RAP) and fly ash as waste that whose is abundant. So interesting to be researched. The research will be started by analyze of $R A P$, be obtainedcharacteristics of aggregates and asphalt, then analyzed on an aggregategradation; if aggregate gradation does not on gradation envelope will be required the addition of new aggregate. If asphaltdoes notmatch the requirements then also be requiredthe addition of new asphalt.The next will be made the composition of mixdesignforAsphalt Concrete - Binder Course (AC-BC) with variations addition of fly ash volume as filler. The aim of research to get variations addition of fly ash volume $(4 \%, 5 \%$ and 6\%)
\end{abstract}

Keywords - Asphalt Concrete - Binder Course (ACBC), Reclaimed Asphalt Pavement (RAP), fly ash.

\section{I.INTRODUCTION}

The material potential from Cold Milling Machine known as Reclaimed Asphalt Pavement (RAP) as the material of the National Road Maintenance in East Java has enough large, $\pm 50.000 \mathrm{~m}^{3}$ every year RAP cointainedasphaltandaggregate. Its can be reused as ubstitution of new asphalt and aggregate in road pavement. The material recycling of RAP can save natural resources (aggregate, sand and asphalt) and reduce the rate environmental damage caused by mining ( Hassan , 2009)

Fly ash is coal waste that whose is abundant, the number of fly ash wasbe estimated as 13 million tonin2015.

The aim this researchis optimalized the usage of fly ash for Reclaimed Asphalt Pavement (RAP), Asphalt Concrete - Binder Course (ACBC) filler.

\section{II.METHOD}

The steps of this researches are:

1. Analysis RAP to getcharacteristics of aggregates and asphalt, then analyzed on an aggregate gradation, if aggregate gradation does not on gradation envelope will be required the addition of new material (aggregate and asphalt).

2. If asphaltdoes notmatchthe requirements then also be requiredthe addition of new asphalt.

3. Make the composition of mix- designforAsphalt Concrete - Binder Course (AC-BC) with variations addition of fly ash volume as filler.

${ }^{1}$ Ratna Handayani, Ria Asih Aryani, Herry Budianto, and Januart Jaya Ekaputri are with Departement of Civil Engineering, Institut Teknologi Sepuluh Nopember, Surabaya, 60111, Indonesia. E-mail: ratnay1999@gmail.com; budiantoherry@yahoo.com; januarti_je@yahoo.com.

\section{RESULTS AND DISCUSSION}

The result of the research are :

A. Characteristics of RAP (aggregate and asphalt )and Characteristics ofnewmaterial (agregat dan aspal).

TABLE 1 .

RESULT THAT ACCORDING TO REQUIREMENT

\begin{tabular}{clcc}
\hline \hline $\begin{array}{c}\text { Characteristic of } \\
\text { rap (agregat)No }\end{array}$ & Type of test & Requirement & Result \\
\hline 1 & Soudness (\%) & Max 12 & 3,15 \\
2 & $\begin{array}{l}\text { Abrasi (\%) } \\
\text { Stickness } \\
\text { aggregat to } \\
\text { aspal } \\
\text { Specific } \\
\text { gravity } \\
\text { gr/cm3 }\end{array}$ & Max.40 & 20,19 \\
Water \\
absorption \\
$(\%)$
\end{tabular}

Table 1 shows that all of resultthat according to requirement at Spesifikasi Binamarga 2010 Revisi 3 Figure 1 shows that sieve size 1/2and sieve size $3 / 8$ out layer from gradation envelope so it does not meet the requirements spesifikasi Binamarga 2010 Revisi 3. In order to meet requirements its,it is necessary to add new material.

Table 2 shows that all of resultthat according to requirement at Spesifikasi Binamarga 2010 Revisi 3 , except in test of penetrasi with result is $49 \mathrm{~mm}$, while required that $(60-70) \mathrm{mm}$. Which on requirement $(60-$ 70) $\mathrm{mm}$. Table 3 shows that all of resultthat according to requirement at Spesifikasi Binamarga 2010 Revisi 3.

Figure 2 shows gradation for Coarse aggregate (CA), Medium aggregate (MA) and Fine aggregate (FA) that will be used as additional materialatRAP (aggregate) so the combined aggregate gradation can be included in "the envelope gradations" that according Spesifikasi Binamarga 2010 revisi 3.

By comparing the physical properties of the new material on the specifications, so it can be concluded that characteristic of aggregates from Asphalt Mixing Plant (AMP) PT surya Marga Utama (SMU) in Pasuruan and Asphalt Pen 60/70 meets the required spesifications so it can be usedasmixture of Asphalt Concrete Binder Course (AC-BC).

The composition of mix- designforAsphalt Concrete Binder Course (AC-BC) with variations addition of fly ash volume as filler.

In order to requireenvelope gradation according to specifications, so new aggregate is added consisting of:
a. Coarse agregat (10-20)
b. Medium agregat(5-10)
c. Fine agregat (0-5) 
The $2^{\text {nd }}$ International Seminar on Science and Technology August $2^{\text {nd }} 2016$, Postgraduate Program Institut Teknologi Sepuluh Nopember, Surabaya, Indonesia

d. Fly ash as filler

B. The first of Combined gradation

Based on gradation of RAP aggregate and new aggregate gradation (CA, MA, FA ) and fly ashso be done the composition of mix design. The details ofits are presented in table 5 and figure 3 .

TABLE 2.

TABLE 3.

CHARACTERISTIC OF RAP (ASPHALT)

\begin{tabular}{cccc}
\hline \hline No & Type of test & Requirement & Result \\
\hline 1 & $\begin{array}{l}\text { Asphalt } \\
\text { content in the } \\
\text { mix }\end{array}$ & - & $4 \%$ \\
& $\begin{array}{l}\text { Penetrasi } \\
\text { pada } 25^{\circ} \mathrm{C} \\
(\mathrm{mm})\end{array}$ & $60-70$ & 49 \\
3 & $\begin{array}{l}\text { Viskositas } \\
135^{\circ} \mathrm{C}(\mathrm{cSt})\end{array}$ & $>300$ & 2.3 \\
4 & $\begin{array}{l}\text { Titik lembek } \\
\left({ }^{\circ} \mathrm{C}\right)\end{array}$ & $>48$ & 55 \\
5 & $\begin{array}{l}\text { Daktilitas } \\
\text { pada } 25^{\circ} \mathrm{C}, \\
(\mathrm{cm})\end{array}$ & $>100$ & $>120$ \\
6 & $\begin{array}{l}\text { Specific } \\
\text { gravity } \\
(\mathrm{gr} / \mathrm{cm} 3)\end{array}$ & $>1,0$ & 1,067 \\
\hline \hline
\end{tabular}

CHARACTERISTIC OF NEW AGREGAT

\begin{tabular}{cllc}
\hline \hline No & Type of test & Requirement & Result \\
\hline 1 & Soudness (\%) & Mak 12 & 8,04 \\
2 & Abrasi(\%) & Mak.40 & 15,91 \\
& $\begin{array}{l}\text { Stickness } \\
\text { aggregat to } \\
\text { aspal }\end{array}$ & Min 95 & $>95$ \\
& $\begin{array}{l}\text { Specific } \\
\text { gravity } \\
\text { gr/cm3 }\end{array}$ & - & \\
& $\begin{array}{l}\text { Water } \\
\text { absorption } \\
(\%)\end{array}$ & - & 2,84 \\
5 & & 1,26 \\
\hline \hline
\end{tabular}

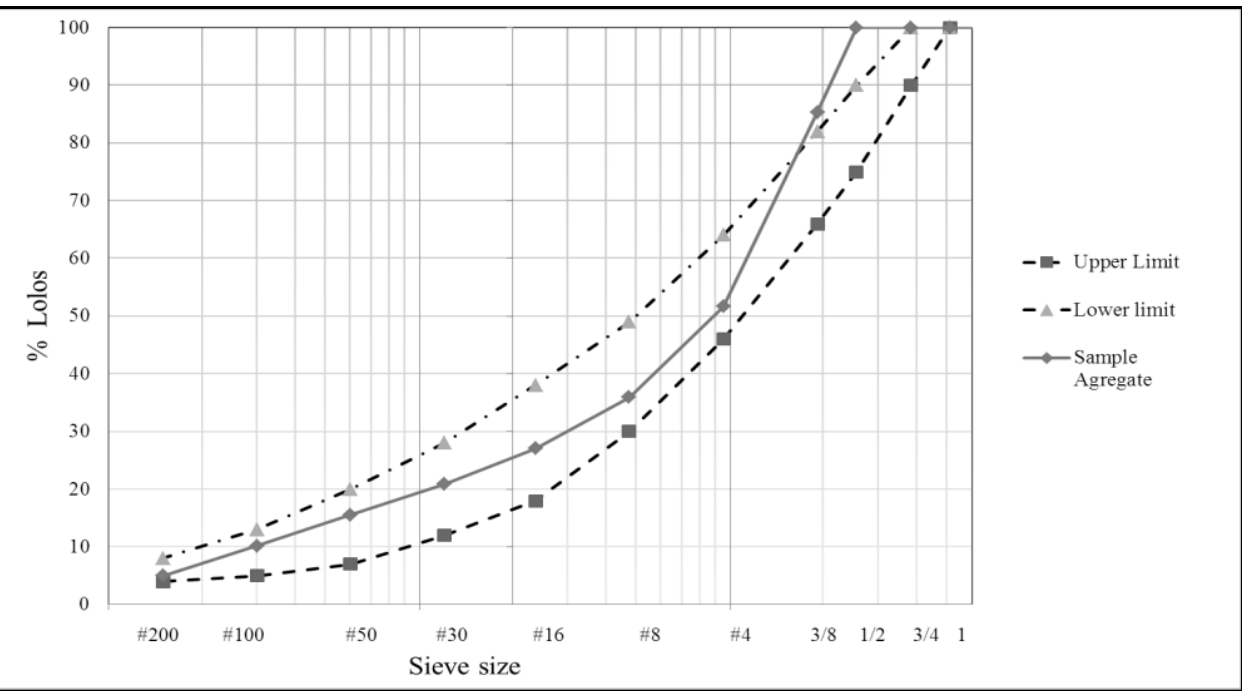

Figure 1. Gradation of RAP Agregat

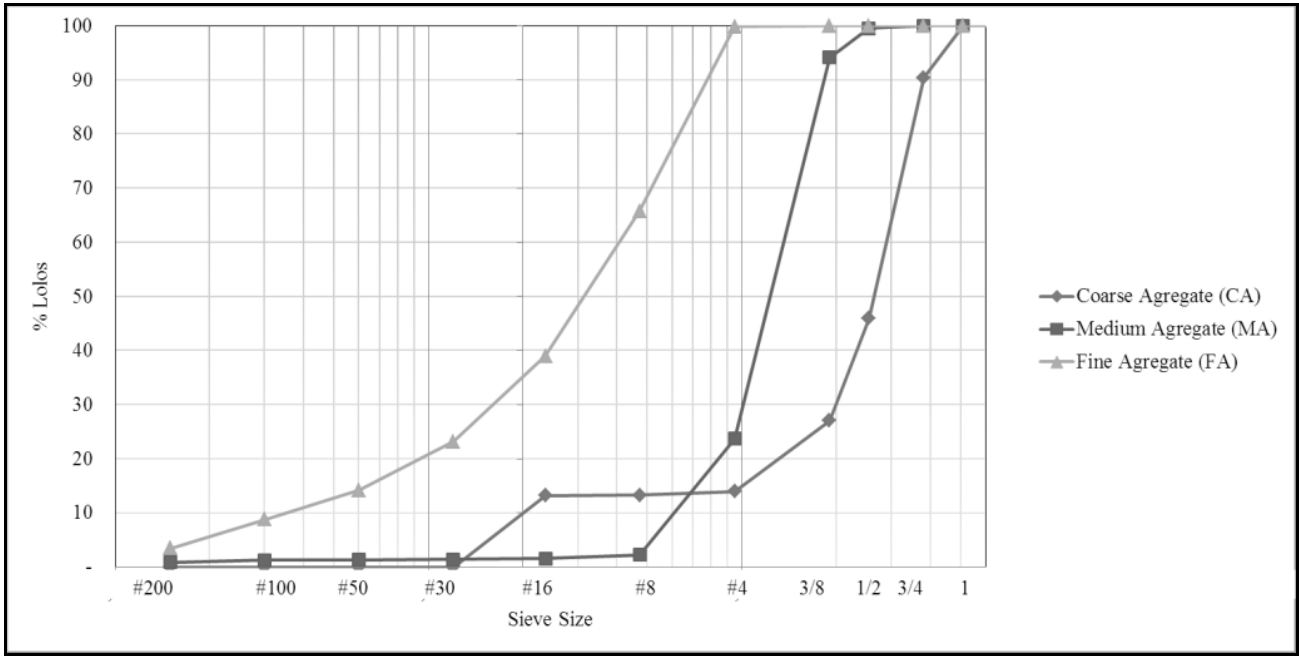

Figure 2. Gradation ofNewAgregat 
The $2^{\text {nd }}$ International Seminar on Science and Technology

August $2^{\text {nd }} 2016$, Postgraduate Program Institut Teknologi Sepuluh Nopember, Surabaya, Indonesia

TABLE 4.

CHARACTERISTIC OF NEW ASPHALT

\begin{tabular}{cccc}
\hline \hline No & Type of test & Requirement & Result \\
\hline 1 & Penetrasi at $25^{\circ} \mathrm{C}(\mathrm{mm})$ & $60-70$ & 62 \\
2 & Viskositas $135^{\circ} \mathrm{C}(\mathrm{cSt})$ & $\geq 300$ & 393 \\
3 & Titik Lembek $\left({ }^{\circ} \mathrm{C}\right)$ & $\geq 48$ & 49,1 \\
4 & Daktilitas pada $25^{\circ} \mathrm{C}(\mathrm{cm})$ & $\geq 100$ & $>140$ \\
5 & Titik Nyala $\left({ }^{\circ} \mathrm{C}\right)$ & $\geq 232$ & 240 \\
6 & SolubilityTrichloroethylene & $\geq 99(\%)$ & 99,82 \\
7 & Specific gravity $(\mathrm{gr} / \mathrm{cm} 3)$ & $\geq 1,0$ & 1,03 \\
& Residu Test of TFOT Result & 0,21 \\
8 & Weight Loss $(\%)$ & $\leq 0.8$ & $\%$ \\
9 & Penetrasi at $25^{\circ} \mathrm{C}(\mathrm{mm})$ & $\geq 54$ & $\%$ \\
10 & Daktilitas pada $25^{\circ} \mathrm{C}(\mathrm{cm})$ & $\geq 100$ & 100 \\
\hline \hline
\end{tabular}

TABLE 5 .

COMBINED AGGREGATE GRADATION WITHFLY ASH OF4 \%

\begin{tabular}{|c|c|c|c|c|c|c|c|c|c|c|c|}
\hline \multirow{3}{*}{$\begin{array}{l}\text { Explanation } \\
\text { Inch }\end{array}$} & \multirow{2}{*}{$\begin{array}{c}\text { Proporsition } \\
(\%)\end{array}$} & \multicolumn{10}{|c|}{ Sieve Size } \\
\hline & & \multicolumn{10}{|c|}{ (\% Lolos) } \\
\hline & & $3 / 4 "$ & $1 / 2 "$ & 3/8" & \#4 & \#8 & \#16 & \#30 & \#50 & \#100 & $\# 200$ \\
\hline $\mathrm{Mm}$ & & 19.0 & 12.5 & 9.5 & 4.75 & 2.36 & 1.18 & 0.600 & 0.300 & 0.150 & 0.075 \\
\hline \multicolumn{12}{|l|}{$\begin{array}{l}\text { Data of } \\
\text { Aggregate } \\
\text { Grading }\end{array}$} \\
\hline - RAP & & 100 & 100 & 85,34 & 51,64 & 35,91 & 27,08 & 20,88 & 15,56 & 10,18 & 5,00 \\
\hline$-\mathrm{CA}$ & & 90,43 & 45,89 & 27,02 & 13,98 & 13,27 & 13,18 & 0 & 0 & 0 & 0 \\
\hline - MA & & 100 & 99,55 & 94,12 & 23,70 & 2,26 & 1,56 & 1,41 & 1,33 & 1,26 & 0,85 \\
\hline - FA & & 100 & 100 & 100 & 99,88 & 65,80 & 38,99 & 23,19 & 14,19 & 8,78 & 3,49 \\
\hline - Fly Ash & & 100 & 100 & 100 & 100 & 100 & 100 & 100 & 100 & 100 & 100 \\
\hline \multicolumn{12}{|l|}{$\begin{array}{l}\text { Agregat } \\
\text { Combinated }\end{array}$} \\
\hline - RAP & 20 & 20 & 20 & 17,07 & 10,33 & 7,18 & 5,42 & 4,18 & 3,11 & 2,04 & 1,00 \\
\hline - CA & 20 & 18,09 & 9,18 & 5,40 & 2,80 & 2,65 & 2,64 & - & - & - & - \\
\hline - MA & 31 & 31,00 & 30,86 & 29,18 & 7,35 & 0,70 & 0,48 & 0,44 & 0,41 & 0,39 & 0,26 \\
\hline - FA & 25 & 25,00 & 25,00 & 25,00 & 24,97 & 16,45 & 9,75 & 5,80 & 3,55 & 2,20 & 0,87 \\
\hline - Fly Ash & 4 & 4,00 & 4,00 & 4,00 & 4,00 & 4,00 & 4,00 & 4,00 & 4,00 & 4,00 & 4,00 \\
\hline Total of mix & 100 & 98,09 & 89,04 & 80,65 & 49,44 & 30,99 & 22,28 & 14,41 & 11,07 & 8,62 & 6,14 \\
\hline \multicolumn{12}{|l|}{$\begin{array}{l}\text { Gradation } \\
\text { Envelope Spek. }\end{array}$} \\
\hline Maks. & & 100 & 90 & 82 & 64 & 49 & 38 & 28 & 20 & 13 & 8 \\
\hline Min & & 90 & 75 & 66 & 46 & 30 & 18 & 12 & 7 & 5 & 4 \\
\hline Ideal Gradation & & 95,0 & 82,5 & $\mathbf{7 4 , 0}$ & 55,0 & 39,5 & 28,0 & 20,0 & 13,5 & $\mathbf{9 , 0}$ & 6,0 \\
\hline
\end{tabular}


The $2^{\text {nd }}$ International Seminar on Science and Technology August $2^{\text {nd }} 2016$, Postgraduate Program Institut Teknologi Sepuluh Nopember, Surabaya, Indonesia

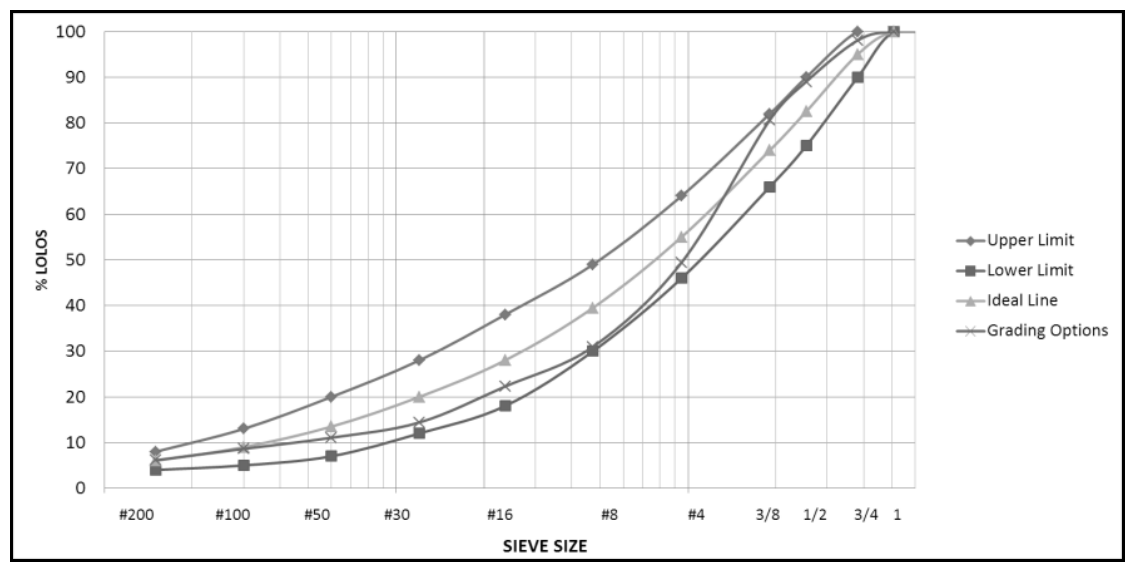

Figure 3. combined aggregate gradation withflyashof $4 \%$

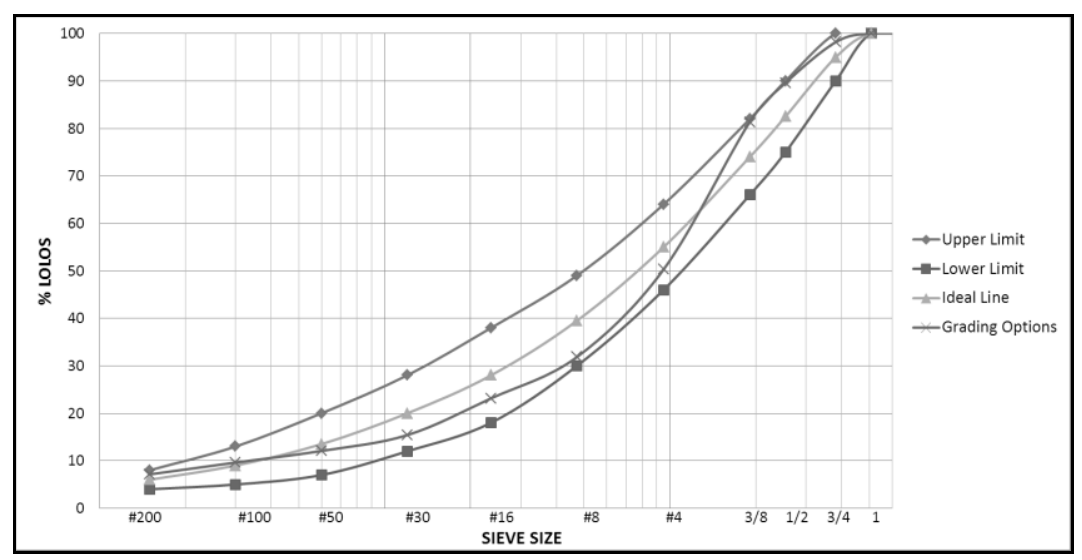

Figure 4. Combined Aggregate Gradation withFly Ashof5 \%

TABLE 6.

COMBINED AGGREGATE GRADATION WITHFLY ASH OF5 \%

\begin{tabular}{|c|c|c|c|c|c|c|c|c|c|c|c|}
\hline \multirow{2}{*}{ Explanation } & Proporsition & \multicolumn{10}{|c|}{ Sieve Size } \\
\hline & $(\%)$ & \multicolumn{10}{|c|}{ (\% Lolos) } \\
\hline Inch & & $3 / 4 "$ & $1 / 2 "$ & $3 / 8$ & $\# 4$ & $\# 8$ & $\# 16$ & $\# 30$ & $\# 50$ & $\# 100$ & \#200 \\
\hline $\mathrm{Mm}$ & & 19.0 & 12.5 & 9.5 & 4.75 & 2.36 & 1.18 & 0.600 & 0.300 & 0.150 & 0.075 \\
\hline \multicolumn{12}{|l|}{$\begin{array}{l}\text { Data of } \\
\text { Aggregate } \\
\text { Grading }\end{array}$} \\
\hline - RAP & & 100 & 100 & 85,34 & 51,64 & 35,91 & 27,08 & 20,88 & 15,56 & 10,18 & 5,00 \\
\hline$-\mathrm{CA}$ & & 90,43 & 45,89 & 27,02 & 13,98 & 13,27 & 13,18 & $\mathrm{o}$ & 0 & 0 & o \\
\hline - MA & & 100 & 99,55 & 94,12 & 23,70 & 2,26 & 1,56 & 1,41 & 1,33 & 1,26 & 0,85 \\
\hline - FA & & 100 & 100 & 100 & 99,88 & 65,80 & 38,99 & 23,19 & 14,19 & 8,78 & 3,49 \\
\hline - Fly Ash & & 100 & 100 & 100 & 100 & 100 & 100 & 100 & 100 & 100 & 100 \\
\hline \multicolumn{12}{|l|}{$\begin{array}{l}\text { Agregat } \\
\text { Combinated }\end{array}$} \\
\hline - RAP & 20 & 20 & 20 & 17,07 & 10,33 & 7,18 & 5,42 & 4,18 & 3,11 & 2,04 & 1,00 \\
\hline$-\mathrm{CA}$ & 19 & 17,18 & 8,72 & 5,13 & 2,66 & 2,52 & 2,50 & - & - & - & - \\
\hline- MA & 31 & 31,00 & 30,86 & 29,18 & 7,35 & 0,70 & 0,48 & 0,44 & 0,41 & 0,39 & 0,26 \\
\hline - FA & 25 & 25,00 & 25,00 & 25,00 & 24,97 & 16,45 & 9,75 & 5,80 & 3,55 & 2,20 & 0,87 \\
\hline - Fly Ash & 5 & 5,00 & 5,00 & 5,00 & 5,00 & 5,00 & 5,00 & 5,00 & 5,00 & 5,00 & 5,00 \\
\hline Total of mix & 100 & 98,18 & 89,58 & $\mathbf{8 1}, \mathbf{3 8}$ & 50,30 & 31,85 & 23,15 & 15,41 & 12,07 & 9,62 & 7,14 \\
\hline \multicolumn{12}{|l|}{$\begin{array}{l}\text { Gradation } \\
\text { Envelope } \\
\text { Spek. }\end{array}$} \\
\hline Maks. & & 100 & 90 & 82 & 64 & 49 & 38 & 28 & 20 & 13 & 8 \\
\hline Min & & 90 & 75 & 66 & 46 & 30 & 18 & 12 & 7 & 5 & 4 \\
\hline \begin{tabular}{|l|} 
Ideal \\
Gradation \\
\end{tabular} & & 95,0 & 82,5 & $\mathbf{7 4 , 0}$ & 55,0 & 39,5 & 28,0 & 20,0 & 13,5 & $\mathbf{9 , 0}$ & 6,0 \\
\hline
\end{tabular}

Figure 3 shows that the addition of the aggregate with the composition according to table 5 can result the combined aggregate gradation that according to specifications. Based on table 5, the composition of the combined aggregate torequire envelope gradation is RAP (Reclaimed Asphalt Pavement) 20\%, CA (Coarse Aggregate) 20\%, MA (Medium Aggregate) 31\%, FA (Fine Aggregate) $25 \%$ and fly ash $4 \%$. 
August $2^{\text {nd }}$ 2016, Postgraduate Program Institut Teknologi Sepuluh Nopember, Surabaya, Indonesia

\section{The second of combined gradation.}

Based on gradation of RAP aggregate and gradation of new aggregate (CA, MA, FA ) and fly ashso be done the composition of mix design. The details ofits are presented in table 6 and figure 4 . Figure 4 shows that the addition of the aggregate with the composition according to table 6 can result the combined aggregate gradation that according to specifications. Based on table 6 , the composition of the combined aggregate torequire envelope gradation is RAP Reclaimed Asphalt Pavement)20\%, CA ( Coarse Aggregate) 19\%, MA (Medium Aggregate) 31\%, FA (Fine Aggregate) $25 \%$ and fly ash $5 \%$.

TABLE 7.

COMBINED AGGREGATE GRADATION WITHFLY ASH OF6 \%

\begin{tabular}{|c|c|c|c|c|c|c|c|c|c|c|c|}
\hline \multirow{2}{*}{ Explanation } & Proporsition & \multicolumn{10}{|c|}{ Sieve Size } \\
\hline & $(\%)$ & \multicolumn{10}{|c|}{ (\% Lolos) } \\
\hline Inch & & $3 / 4 "$ & $1 / 2 "$ & $3 / 8 "$ & $\# 4$ & $\# 8$ & $\# 16$ & $\# 30$ & \#50 & $\# 100$ & $\# 200$ \\
\hline $\mathrm{Mm}$ & & 19.0 & 12.5 & 9.5 & 4.75 & 2.36 & 1.18 & 0.600 & 0.300 & 0.150 & 0.075 \\
\hline \multicolumn{12}{|l|}{$\begin{array}{l}\text { Data of Aggregate } \\
\text { Grading }\end{array}$} \\
\hline - RAP & & 100 & 100 & 85,34 & 51,64 & 35,91 & 27,08 & 20,88 & 15,56 & 10,18 & 5,00 \\
\hline$-\mathrm{CA}$ & & 90,43 & 45,89 & 27,02 & 13,98 & 13,27 & 13,18 & 0 & 0 & 0 & 0 \\
\hline - MA & & 100 & 99,55 & 94,12 & 23,70 & 2,26 & 1,56 & 1,41 & 1,33 & 1,26 & 0,85 \\
\hline - FA & & 100 & 100 & 100 & 99,88 & 65,80 & 38,99 & 23,19 & 14,19 & 8,78 & 3,49 \\
\hline - Fly Ash & & 100 & 100 & 100 & 100 & 100 & 100 & 100 & 100 & 100 & 100 \\
\hline \multicolumn{12}{|l|}{$\begin{array}{l}\text { Agregat } \\
\text { Combinated }\end{array}$} \\
\hline - RAP & 20 & 20 & 20 & 17,07 & 10,33 & 7,18 & 5,42 & 4,18 & 3,11 & 2,04 & 1,00 \\
\hline$-\mathrm{CA}$ & 28 & 25,32 & 12,85 & 7,57 & 3,91 & 3,72 & 3,69 & - & - & & \\
\hline - MA & 26 & 26,00 & 25,88 & 24,47 & 6,16 & 0,59 & 0,41 & 0,37 & 0,35 & 0,33 & 0,22 \\
\hline - FA & 20 & 20,00 & 20,00 & 20,00 & 19,98 & 13,16 & 7,80 & 4,64 & 2,84 & 1,76 & 0,70 \\
\hline - Fly Ash & 6 & 6,00 & 6,00 & 6,00 & 6,00 & 6,00 & 6,00 & 6,00 & 6,00 & 6,00 & 6,00 \\
\hline Total of mix & 100 & 97,32 & 84,73 & 75,10 & 46,38 & 30,65 & 23,31 & 15,18 & 12,29 & 10,12 & $\mathbf{7 , 9 2}$ \\
\hline \multicolumn{12}{|l|}{$\begin{array}{l}\text { Gradation } \\
\text { Envelope Spek. }\end{array}$} \\
\hline Maks. & & 100 & 90 & 82 & 64 & 49 & 38 & 28 & 20 & 13 & 8 \\
\hline Min & & 90 & 75 & 66 & 46 & 30 & 18 & 12 & 7 & 5 & 4 \\
\hline Ideal Gradation & & 95,0 & 82,5 & 74,0 & 55,0 & 39,5 & 28,0 & 20,0 & 13,5 & 9,0 & 6,0 \\
\hline
\end{tabular}

D. The third of combined gradation

Based on gradation of RAP aggregate and gradation ofnew aggregate (CA, MA, FA ) and fly ashso be done the composition of mix design. The details ofits are presented in table 7 and figure 5 .

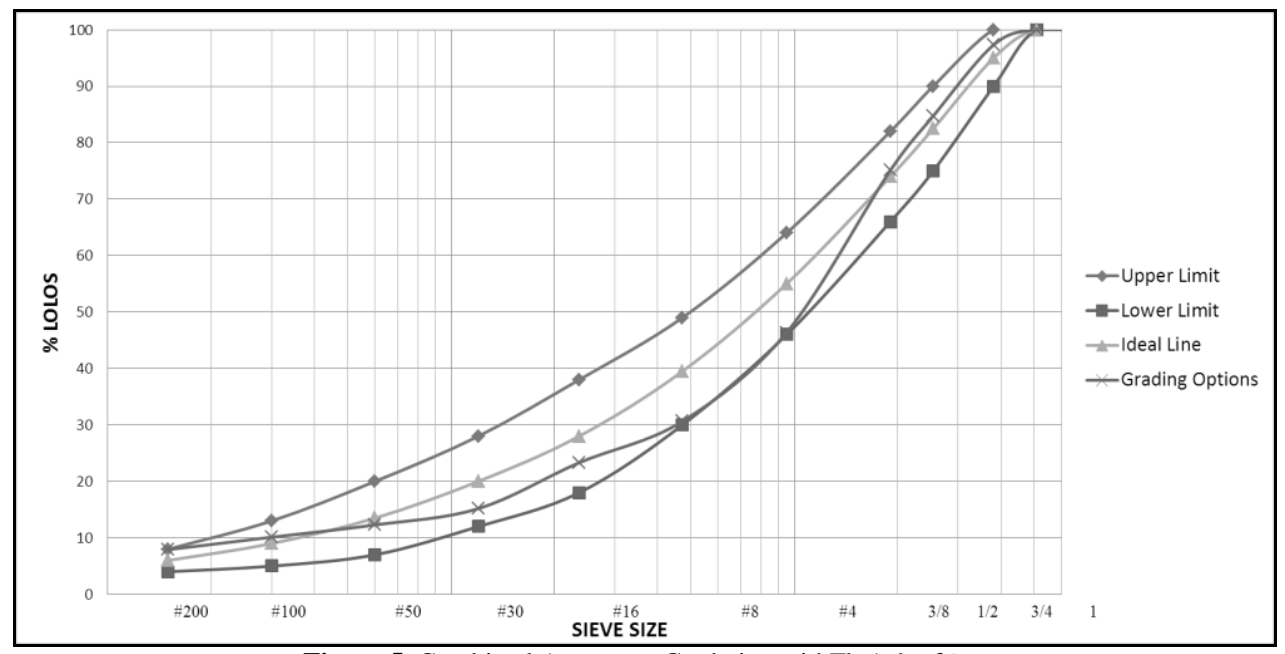

Figure 5. Combined Aggregate Gradation withFlyAsh of $6 \%$

Figure 5 shows that the addition of the aggregate with the composition according to table 7 can result the combined aggregate gradation that according to specifications. Based on table 7, the composition of the combined aggregate torequire envelope gradation is RAP (Reclaimed Asphalt Pavement)20\%, CA (Coarse Aggregate) 28\%, MA (Medium Aggregate) 26\%, FA (Fine Aggregate) $20 \%$ and fly ash 6\%. 
The $2^{\text {nd }}$ International Seminar on Science and Technology August $2^{\text {nd }} 2016$, Postgraduate Program Institut Teknologi Sepuluh Nopember, Surabaya, Indonesia

\section{CONCLUSION}

Result from the usage of fly ash for RAP, AC-BC filler that according to Bina Marga Specification at 2010 Revisi 3 are :

1. The composition of the combined aggregate is RAP (Reclaimed Asphalt Pavement) 20\%, CA (Coarse Aggregate) 20\%, MA (Medium Aggregate) 31\%, FA (Fine Aggregate) $25 \%$ and fly ash $4 \%$.

2. The composition of the combined aggregate is RAP (Reclaimed Asphalt Pavement)20\%, CA ( Coarse
Aggregate) 19\%, MA (Medium Aggregate) 31\%, FA (Fine Aggregate) $25 \%$ and fly ash 5\%.

3. The composition of the combined aggregate is RAP (Reclaimed Asphalt Pavement)20\%, CA (Coarse Aggregate) 28\%, MA (Medium Aggregate) 26\%, FA (Fine Aggregate) $20 \%$ and fly ash $6 \%$.

\section{REFERENCES}

[1] Direktorat Jenderal Bina Marga, Kementrian Pekerjaan Umum, "Spesifikasi Umum Edisi 2010 Revisi 3, 2010.

[2] NAPA Education Foundation, Hot Mix Asphalt Materials Mixture Design and Construction, 1996 